technicians against all work which might be useful in war.

American writing is often turgid and heavy. Dr. Piel, however, has a lucid style and is not without wit. $\mathrm{He}$ is on the side of the angels, even though he rejects their reality. His book throws much light on the American scene, political and educational. His sympathy is broad, and his picture of science as both beautiful and illuminating has its attraction. His book represents the best in American scientific humanism, and opens up problems of the modern age which must engage the attention of all responsible men.

Marcus KNIGHT

\section{THE NATURE OF TIME}

The Natural Philosophy of Time

By Dr. G. J. Whitrow. Pp. xi +324 . (London and Edinburgh: Thomas Nelson and Sons, Ltd., 1961.) 42s. net.

IME is probably the most fundamental observ. able in science, and it is curious that the idea of time is so much taken for granted. We learn how to measure it with remarkable accuracy without concerning ourselves with what it is we are measuring, and very few undergraduate courses in, for example, applied mathematics or physics, deal honestly with questions involving the nature of time. Relativity theory touches them, but even there it is assumed that every observer has a built-in clock about which he does not need to ask questions-one result of which is the now famous clock paradox.

The late E. A. Milne was probably the most eminent theoretical physicist who refused to take time for granted but asked - and to some extent answeredfundamental questions about the role of time in modern science, and since Dr. Whitrow was a student and collaborator of Milne's it is not surprising that it is from him we receive this long overdue account of the subject. Nor is it surprising that the author has devoted more than half the book to the various mathematical problems that arise in the study of primitive clocks and time-scales and the position of time in relativity theory, because it is to this part of the subject that he has made his own major contributions. Nevertheless, two very substantial chapters, of a non-mathematical nature, are given to the ideas and observations contributed by philosophy, psychology and biology to the subject, and in view of the large amount of material collected here it is to the author's credit that he has been able to present it in such an interesting way. A critical account, with adequate references, is given of the various philosophical theories and comments on the nature of time, and the more recent contributions of experimental psychology and biology are described clearly and fitted into an unfolding pattern. Even if the mathematical parts are ignored, the book is well worth reading for these chapters alone.

The remaining four chapters are concerned with the logical and mathematical aspects of the subject, starting in Chapter 3 with a study of the postulates of temporal instants, order and measurement, and going on in Chapters 4 and 5 to the study of time in the mathematical framework of relativity and cosmology. In Chapter 4 there is described a system of eleven axioms following formal definitions of the time and distance assigned by an observer to a distant ovent. The consequences of these are worked out without undue mathematical complication-needing in fact little more than the idea of a continuous function-and the resulting structure is compared with that of Einstein's special theory of relativity and also with that given axiomatically by A. A. Robb. In Chapter 5 there are an account and some discussion of the position of the time parameter in various standard space-times of general relativity and cosmology, and in Chapter 6 there is a brief discussion of the nature of time in modern physics, dealing particularly with asymmetry, the causal and statistical theories, the Minkowski diagram and, finally, precognition. In this second part of the book there is much to interest mathematicians and physicists as well as philosophers, and the whole book should undoubtedly be read by every scientist and natural philosospher. The printing and production are excellent.

A. G. WALKER

\section{HIGH-ENERGY NUCLEAR PHYSICS}

\section{Kinematics of Nuclear Reactions}

By A. M. Baldin, V. I. Goldanskii, and I. L. Rozental'. Translated by Ronald Peierls. Pp. xii $+223+57$ tables. (London: Oxford University Press, 1961.) 38s. net.

A $T$ the heart of every experiment in high-energy A nuclear physics, involving the collision of particles, lie two basic topics, one being the kinematics of the reaction and the other the theoretical interpretation of the reaction. This book, intended by the authors to be, primarily, for experimental physicists, deals with these two subjects. The first half is coneerned with the classical theory of relativistic kinematics and contains the derivation of the mathematical equations required to solve two-body, and, under certain limiting conditions, multi-body kinematics.

This includes such topics as the Lorentz transformation, four-vectors, the Jacobian and other kinematical transformations necessary in going from the laboratory system of co-ordinates into the centre-of-mass system of the two colliding bodies. Decay kinematies of particles which decay into two others are also treated in detail. Appendix 1 contains graphical illustrations of the results of computations based on the formula derived in the first part, and a fair selection of the two-body reactions has been made. One might have included some graphs of decay kinematics, but, obviously, it is not practicable to cover all possibilities and still retain a book of reasonable size.

The second part is concerned with the quantum mechanical aspects of the interactions between particles and requires an introductory course in quantum mechanics for full appreciation and understanding. A discussion on the properties of the scattering $(S)$-matric and its applications is lucid, and topics like diffraction scattering, the optical theorem and the principle of detailed balance are dealt with in a straightforward manner. The chapter dealing with collisions of particles with spin becomes more mathematical in treatment and introduces vector coupling (Clebsch-Gordon) coefficients and the $W, X$ and $Z$ (Racah) coefficients. Other subjects discussed include the phenomenon of polarization and photo-reactions, such as photo-meson production and nucleon Compton scattering. In the second appendix to this part there are some 57 tables of varying complexity giving values of $W, X, Z$, and Clebsch-Gordon coefficients, and the work involved 